\title{
FRACTURE REPAIR
}

\section{Could blockade of Notch signalling promote fracture repair?}

Fracture repair relies on contributions from multiple cell types and signalling pathways, details of which require further clarification. A new study by Matthews et al. has, however, added insight into the involvement of periosteal mesenchymal progenitor cells in this process.

Fluorescent labelling of these progenitor cells based on expression of $\alpha$-smooth muscle actin ( $\mathrm{SSMA}$ ) revealed that their numbers were markedly expanded after induction of fractures in mice. "After fracture, the labelled cells exhibited

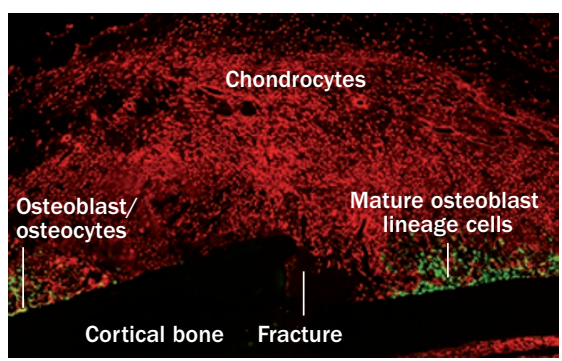

Abundant $a \mathrm{SMA}^{+}$cells (red) contribute to multiple skeleta elements within the fracture callus, including osteoblasts and/or osteocytes (yellow cells) and chondrocytes. Image courtesy of Brya G. Matthews and Ivo Kalajzic. increased expression of genes associated with chemokine or cytokine signalling and the cell cycle," explains corresponding author Ivo Kalajzic. Furthermore, levels of chondrocyte and osteoblast markers were increased in fracture callus $\mathrm{aSMA}^{+}$cells. These findings suggest that $\alpha \mathrm{SMA}^{+}$periosteal progenitor cells might help coordinate the inflammatory phase of fracture healing and represent key precursors of cell types required for repair.

Transcriptome analysis also revealed that components of the Notch signalling pathway were substantially downregulated in $\mathrm{aSMA}^{+}$progenitor cells after fracture, including all Notch receptors and canonical downstream targets. "Notch signalling is an important mediator of communication between neighbouring cells, and controls cell proliferation and differentiation," explains Kalajzic. "Our findings thus indicate that decreased Notch pathway activity might be necessary for the commitment of the progenitor cells into mature lineages."
Matthews and colleagues subsequently evaluated this hypothesis in vitro, showing that constitutive expression of the Notch1 intracellular domain in $\mathrm{SSMA}^{+}$cells almost completely prevented differentiation of periosteal cultures into mature mesenchymal lineage cells. Moreover, activation of Notch signalling decreased the capacity of periosteal cells to form new ossicles when implanted subcutaneously within collagen gels in mice.

"We plan to investigate the effects of forced Notch activation and inhibition of Notch signalling during various stages of the repair process in vivo, to determine how modulating this pathway in progenitor cell populations affects fracture healing," Kalajzic concludes.

David Killock

\footnotetext{
Original article Matthews, B. G. et al. Analysis of aSMAlabeled progenitor cell commitment identifies Notch signaling as an important pathway in fracture healing. J. Bone Miner. Res. doi:10.1002/jbmr.2140
} 